Spatially resolved Raman spectroscopy on indium-catalyzed core-shell germanium nanowires: size effects

This article has been downloaded from IOPscience. Please scroll down to see the full text article.

2010 Nanotechnology 21105703

(http://iopscience.iop.org/0957-4484/21/10/105703)

View the table of contents for this issue, or go to the journal homepage for more

Download details:

IP Address: 128.178.106.14

The article was downloaded on 02/01/2012 at 16:06

Please note that terms and conditions apply. 


\title{
Spatially resolved Raman spectroscopy on indium-catalyzed core-shell germanium nanowires: size effects
}

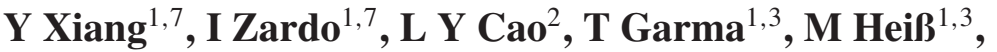 \\ J R Morante M $^{4,5}$, J Arbiol ${ }^{4,6}, \mathbf{M}_{\text {L }}$ Brongersma $^{2}$ and \\ A Fontcuberta i Morral ${ }^{1,3}$ \\ ${ }^{1}$ Walter Schottky Institut, Physik Department, Technische Universitaet Muenchen, \\ Am Coulombwall 3, D-85748 Garching, Germany \\ ${ }^{2}$ Geballe Laboratory for Advanced Materials, 476 Lomita Mall, Stanford University, Stanford, \\ CA 94305, USA \\ ${ }^{3}$ Laboratoire des Matériaux Semiconducteurs, Institut des Matériaux, Ecole Polytechnique \\ Fédérale de Lausanne, CH-1015 Lausanne, Switzerland \\ ${ }^{4}$ Departament d'Electronica, Universitat de Barcelona, 08028 Barcelona, CAT, Spain \\ ${ }^{5}$ IREC, Catalonia Institute for Energy Research, Barcelona 08019, CAT, Spain \\ ${ }^{6}$ ICREA Research Professor at Institut de Ciència de Materials de Barcelona, CSIC, 08193 \\ Bellaterra, CAT, Spain
}

Received 21 August 2009, in final form 10 November 2009

Published 15 February 2010

Online at stacks.iop.org/Nano/21/105703

\begin{abstract}
The structure of indium-catalyzed germanium nanowires is investigated by atomic force microscopy, scanning confocal Raman spectroscopy and transmission electron microscopy. The nanowires are formed by a crystalline core and an amorphous shell. We find that the diameter of the crystalline core varies along the nanowire, down to few nanometers. Phonon confinement effects are observed in the regions where the crystalline region is the thinnest. The results are consistent with the thermally insulating behavior of the core-shell nanowires.
\end{abstract}

(Some figures in this article are in colour only in the electronic version)

One-dimensional materials such as nanowires are promising building blocks for future nanoscale device applications such as biological/chemical sensors, high mobility transistors and solar cells [1-4]. Additionally, semiconductor nanowires provide a resource-filled platform for fundamental condensed matter research [5-8]. Among the various semiconductors obtained in the form of nanowires, special attention has been given to germanium nanowires $[9,10]$. In comparison with silicon, the most widely used material in electronics, germanium has small effective electron and hole masses, that lead to higher carrier mobility. Indeed, germanium nanowire field effect devices may exhibit better performance than planar and nanowire silicon technology. Moreover, germanium nanowires can be grown on silicon substrates, thereby guaranteeing integration with silicon technology [11-13].

The most commonly used method for the synthesis of nanowires is the vapor-liquid-solid method (VLS), in which a

7 Equal contribution. liquid metal nanoscale droplet is responsible for the nucleation and growth of the nanowires. The most common metal used is gold. Incorporated in the matrix, gold forms a deep level in the bandgap and is responsible for the reduction of lifetime of the carriers $[14,15]$. It is for this reason that the use of alternative catalysts such as aluminum, indium, bismuth and gallium has been investigated [14, 16-20]. These metals are slightly less soluble than gold in silicon and germanium and at the same time do not form deep level traps [21].

In this work, we investigate the structural properties of indium-catalyzed germanium nanowires. The synthesis was realized in a chemical vapor deposition furnace. Details of the growth system can be found elsewhere [19]. The substrates were fused quartz wafers with $5 \mathrm{~nm}$ of In, which was deposited by electron beam evaporation. Prior to the nanowire growth, a 15 min annealing was performed at $800{ }^{\circ} \mathrm{C}$ under a flow of $100 \mathrm{sccm} \mathrm{H}_{2}$ and a pressure of 30 Torr in order to reduce the oxidized indium film [19]. For the nanowire synthesis, the temperature of the furnace was lowered to $300^{\circ} \mathrm{C}$. A mixture 
a)

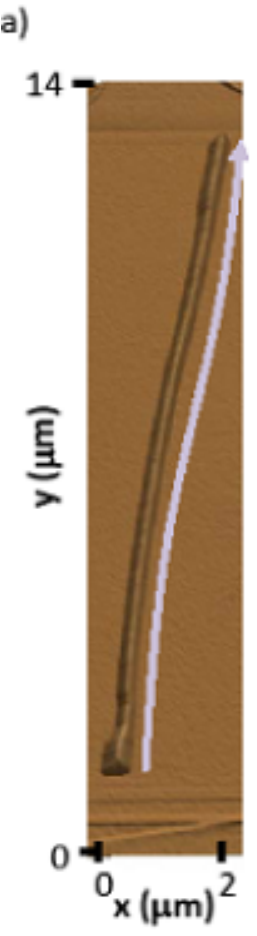

b)

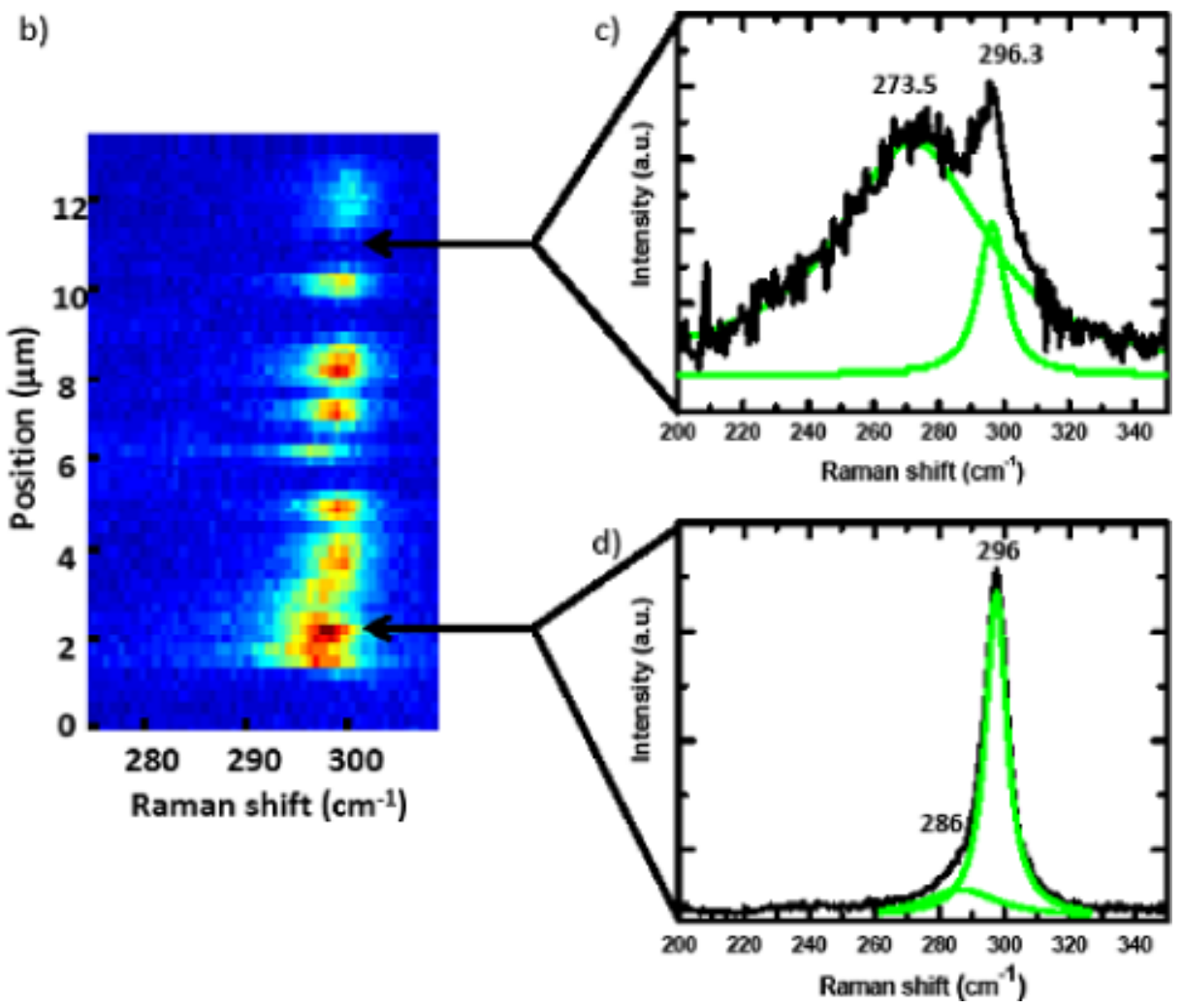

Figure 1. (a) AFM image of a single nanowire. The arrow represents the scanning direction during the Raman measurements. (b) One-dimensional Raman color plot interpolated obtained from a 2D Raman mapping of a single germanium nanowire. Details of the spectra in the specified positions are shown in (c) and (d). The continuous lines underneath the measured spectra are Lorentzian peaks with which the spectra have been analyzed.

of $10 \%$ germane in argon was introduced into the growth chamber. The nanowires studied in this work correspond to the ones exhibiting the best aspect ratios-least tapering - and highest density on the substrate. The diameters are found to be between 70 and $200 \mathrm{~nm}$.

The morphology and structure of the nanowires was characterized by atomic force microscopy (AFM), Raman spectroscopy and transmission electron microscopy (TEM) in bright field (BF) and high resolution (HR) modes as well as scanning TEM (STEM) in annular dark field mode (ADF). For AFM and Raman spectroscopy measurements the nanowires were transferred mechanically from the as grown sample to a patterned silicon substrate. Patterning of the substrate enabled the identification of the nanowires for multiple measurements. For TEM investigations, the nanowires were transferred to a holey carbon copper grid as reported elsewhere [22].

Prior to the Raman spectroscopy measurements, the size and morphology of the nanowires was measured by AFM. An example is given in figure 1(a). The diameter of the nanowire, as obtained from the height, varied from 250 to $200 \mathrm{~nm}$ along the nanowire. This confirms the slightly tapered geometry of the nanowires, as observed previously by SEM. To gain insight into the structural variations along the nanowire, confocal Raman microscopy was realized on the same nanowire along the growth axis. The excitation wavelength was the $514.5 \mathrm{~nm}$ line of an $\mathrm{Ar}^{+}$laser. The laser was focused on the nanowire with a $100 \times$ objective (NA 0.95 ), leading to a laser spot size of about $600 \mathrm{~nm}$. The power of the excitation was about
$200 \mu \mathrm{W}$ (equivalent to $70 \mathrm{~kW} \mathrm{~cm}{ }^{-2}$ ), in order to avoid heating of the nanowire [23-25]. A series of measurements with increasing laser power enabled us to understand the power at which heating effects appear-heating effects are characterized by a frequency downshift and broadening of the phonon peaks. The scattered light was collected by an $X Y$ Raman Dilor triple spectrometer with a multichannel charge coupled device detector. The sample was positioned on an $X Y$ piezostage, which allowed the scanning of the surface with a precision of $10 \mathrm{~nm}$.

A typical Raman scattering map of a $12 \mu \mathrm{m}$ long nanowire is shown in figure 1(b). It is clear that the Raman spectrum varies strongly along the nanowire. Two main characteristics are observed in figure 1(b): (i) intensity variations along the nanowire axis and (ii) recurrent shifting of the degenerate TO/LO phonon peak along the axis. We start by commenting on the variations in the intensity. Very sharp variations in intensity are observed in the second top part of the nanowire spectrum. The sharp reduction in intensity could be related to the discontinuity of the nanowire-absence of material. However, AFM measurements indicate that the nanowire is continuous and that the diameter is relatively constant. In order to better understand the decrease in scattering intensity, single spectra were obtained in the distinct regions. Spectra in sections of the nanowire with a high and low scattering intensity were obtained by increasing the integration time by a factor of 4 . These are shown in figures $1(\mathrm{c})$ and (d), with the contributions resulting from 


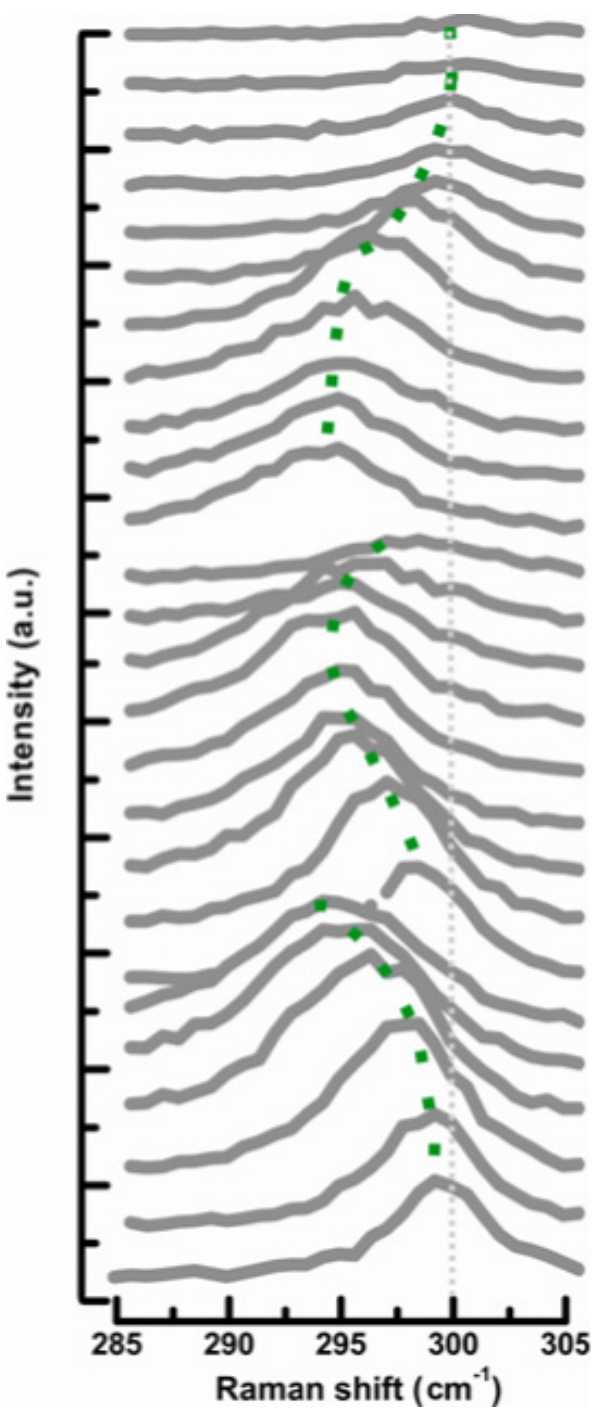

Figure 2. Raman waterfall plot. The Raman spectra were taken every $100 \mathrm{~nm}$. The integration time was $240 \mathrm{~s}$. As a guide to the eye, the light gray dashed line indicates the position of the degenerated $\mathrm{Ge} \mathrm{TO} / \mathrm{LO}$ mode, while the green dashed line indicates the evolution of the actual position of the Raman mode

a multiple Lorentzian fit. As indicated by the presence of a broad peak centered at $273 \mathrm{~cm}^{-1}$ and a sharp peak at $296.5 \mathrm{~cm}^{-1}$, the Raman spectrum corresponding to the dark regions in figure 2(b) is typical of a material with a very large ratio of amorphous to crystalline-figure 1(c). Namely, the amorphous germanium gives a broad peak centered around $\sim 280 \mathrm{~cm}^{-1}$, while the crystalline germanium has a TO/LO phonon mode at $298 \mathrm{~cm}^{-1}[26,19]$. The Raman spectrum of the 'bright' regions is mainly composed of a sharp peak close to $296 \mathrm{~cm}^{-1}$. These results are in agreement with the existence of a crystalline-amorphous core-shell structure, as we have previously reported [19]. Detailed TEM analysis of the nanowire structure will be presented later in the manuscript.

The crystalline fraction $\boldsymbol{F}_{\mathrm{c}}$ in a material has sometimes been estimated by integrating the peak intensity associated with the amorphous and crystalline phases, $\boldsymbol{I}_{\mathrm{a}}$ and $\boldsymbol{I}_{\mathrm{c}}$ respectively [27]:

$$
F_{\mathrm{c}}=\frac{I_{\mathrm{c}}}{I_{\mathrm{c}}+y I_{\mathrm{a}}}
$$

where $y$ corresponds to the scattering cross-section ratio between the crystalline and amorphous phases. For macroscopic samples, the value of $y$ lies between 0.1 and 0.9 , which is mainly due to the difference in the coefficient of absorption at the incident illumination energy [28]. However, for samples containing crystalline sizes of $100 \mathrm{~nm}$ or lower, it has been shown that $y$ may vary significantly [29]. Moreover, $y$ depends on the structural quality of the amorphous layer, as the Raman scattering cross-section increases with density [30]. We have calculated the ratio $100 I_{\mathrm{c}} / I_{\mathrm{c}}+I_{\mathrm{a}}$ along the nanowire, which would correspond to the crystalline fraction if $y=1$. The ratio varies from nearly $100 \%$ down to $8 \%$ in certain regions of the nanowire. This is an extremely large variation, which is certainly related to a dramatic change in the crystalline fraction. It certainly has important consequences for the electronic transport properties.

In addition to the variation in the crystalline fraction, a recurrent shift towards lower frequencies is observed. A shift to lower frequencies is often related to quantum confinement effects $[31,32]$. Our extensive TEM analysis established that the diameter of the core is smaller for nanowires with smaller diameter [19]. In order to decide whether the observed shift is due to quantum confinement effects in a clearer way, a thinner germanium nanowire was investigated. The diameter was $86 \mathrm{~nm}$, as determined by AFM. The Raman spectra of nanowire regions spaced $100 \mathrm{~nm}$ apart are presented in a cascade plot in figure 2. Only the peak corresponding to the TO/LO phonon mode of crystalline germanium is observed. The absence of the amorphous peak signal could be due to various effects: (i) the small integration time used for the measurement only leads to the recording of the predominant feature, (ii) due to the small diameter of the nanowire, the fraction of amorphous material is significantly smaller than for a larger diameter and/or (iii) the density of the amorphous shell is smaller than that of the crystalline core. In figure 2, the position of the unstrained crystalline germanium is plotted with a discontinuous line as a reference. Along the $2.6 \mu \mathrm{m}$ of the scan, a shift of the peak towards lower frequencies is observed. The shift of the Raman spectra could be attributed to the phonon confinement in the core of the germanium nanowires. Indeed, the active Raman mode of crystalline germanium is found only at the zone center $(q=0)$, due to the conservation of phonon momentum. In amorphous germanium, this rule does not apply, meaning that the Raman spectrum is much broader and reflects the phonon density of states [31]. When the dimensions of crystalline germanium are reduced, the phonon is restricted to the volume of the crystal. This leads to a relaxation of the momentum conservation rule, which leads to the broadening and downshift of the peak [31]. In order to obtain the crystalline size from the Raman lines, the peaks should be fitted following the function [32]

$$
I(\omega) \cong \int \frac{\mathrm{d}^{3} q|C(0, q)|^{2}}{(\omega-\omega(q))^{2}+\left(\Gamma_{o} / 2\right)^{2}}
$$



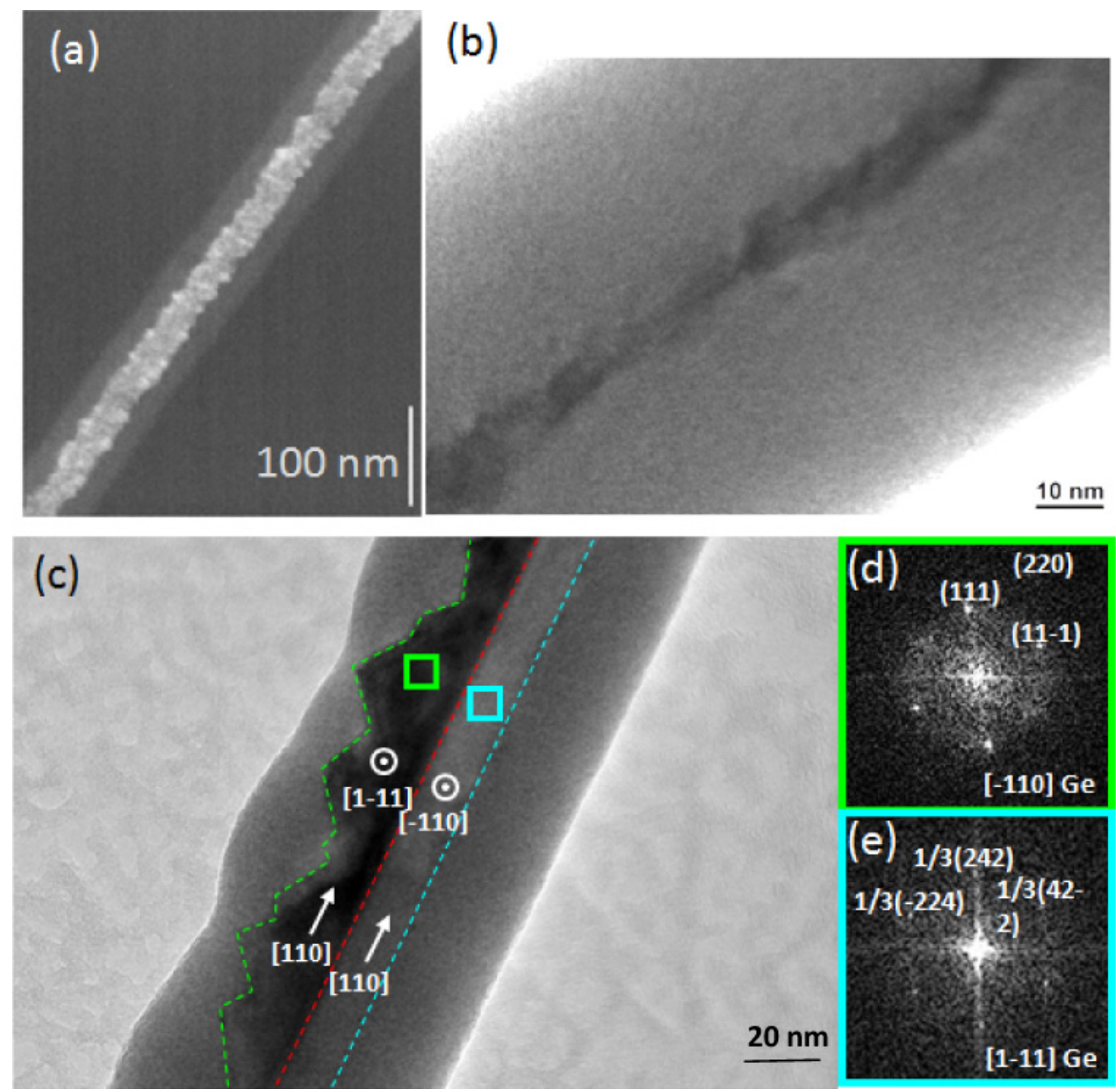

Figure 3. (a) STEM annular dark field (ADF) micrograph obtained in one part of a long nanowire, showing a 40 nm crystalline core capped with a $21 \mathrm{~nm}$ amorphous layer. (b) Bright field TEM micrograph of another nanowire, where the crystalline core (in darker contrast) has shrunk to $5 \mathrm{~nm}$, while the shell continues to be about $40 \mathrm{~nm}$. (c) HRTEM micrograph of a NW with a rough bicrystalline core and amorphous shell. (d) and (e) correspond to the power spectra obtained on the green and blue squared regions in (c), respectively.

where $C(0, q)$ are the Fourier coefficients corresponding to the nanocrystal phonon wavefunction, also called the weighting function. In some models $C(0, q)$ is chosen as a Gaussian weighting function, while in others a sinc weighting function is preferred. The latter would be better related to the ground state of an electron in a sphere $[32,33]$. Our data fit quite nicely with the model in which the nanocrystal is assumed to have a spherical shape. According to this, the observed downshift of the TO/LO phonon mode of $6 \mathrm{~cm}^{-1}$ would correspond to a crystal size smaller than $30 \mathrm{~nm}$. One should add that the contribution of strain to the peak downshift cannot be excluded, since the broadening of the peak is quite small. The spatially resolved Raman scattering measurements indicate a variation of the core diameter along the nanowire.

In order to contrast the information deduced by Raman scattering, TEM measurements were realized. Figure 3(a) corresponds to a STEM annular dark field (ADF) micrograph obtained in one part of a long nanowire, with a diameter of approximately $82 \mathrm{~nm}$. ADF STEM micrographs enable us to discern between crystalline and amorphous regions, as highest intensity areas are mainly obtained when coherent scattered electrons (electrons diffracted under Bragg conditions) reach the annular detector. Interestingly, a $40 \mathrm{~nm}$ crystalline core capped with a $21 \mathrm{~nm}$ amorphous layer is observed. Electron energy loss spectroscopy and high angle annular dark field (HAADF) STEM measurements indicated that both core and shell are pure germanium, not shown here, but demonstrated in a previous work [19]. The oxide is limited to the $2 \mathrm{~nm}$ of the nanowire surface. The core is visibly corrugated, indicating that the monocrystalline core has an inhomogeneous morphology. In agreement with the Raman measurements, the morphology of the crystalline core is inhomogeneous along the axis. Indeed, in figure 3(b) a bright field TEM micrograph of another nanowire is shown. There, the crystalline core (in darker contrast) has shrunk down to $5 \mathrm{~nm}$, while the shell continues to be about $40 \mathrm{~nm}$. The inhomogeneous morphology 
of the core can be better appreciated in figure 3(c), where a HRTEM micrograph of another In-catalyzed Ge nanowire is shown. In this case the core is bicrystalline: the core is divided into two regions separated by a perfectly flat grain boundary. On the left side of figure 3(c), a rough monocrystalline domain is observed, as marked between the green and red dashed lines. This left side core domain is flat on the right grain boundary side, while it has saw-tooth morphology on the left side on the boundary with the amorphous shell. Annexed to this, on the right side between the red and cyan dashed lines, one finds a smooth single crystal. Interestingly enough, the two crystals exhibit the same crystalline [110] growth axis, but with an axially rotated grain boundary (marked with a red dashed line in the above HRTEM micrograph). For clarity, the surface of the two crystals has been indicated with green and blue dashed lines, while the grain boundary has been marked with a red dashed line. The crystalline structure of the two crystals was analyzed by power spectra obtained on the HRTEM images. Both crystals grow along the [110] axis. However, the left side is oriented along the [111] zone axis while the right one along the [110] zone axis, corresponding to a rotation of $35.26^{\circ}$ along the nanowire growth axis. The rough side of the core presents an inhomogeneous thickness, ranging from 10 to $26 \mathrm{~nm}$, and presenting a sort of saw-tooth morphology. We do not detect any stress in the crystalline core. However, the core diameter changes significantly along the nanowire. The shrinking of the crystalline core might pose a problem in the attempt of using these nanowires for electronic devices, as they currently may be stopped (open circuit).

Finally, we would like to mention that we have tried to characterize the electric properties of these germanium nanowires. For this, metallic contacts were fabricated by following a standard process based on electron beam lithography [34]. Two types of contact were realized: (i) directly on the nanowire surface after etching the natural oxide and (ii) directly on the nanowire core by etching the natural oxide and the amorphous core. The metal used was titanium/indium/gold. The contacts were separated by a distance of about $1 \mu \mathrm{m}$. Several contacts were fabricated on each nanowire. In all cases, the resistance was higher than $1 \mathrm{G} \Omega$-reaching the limit of our measurement set-up [35]. We attribute the absence of current to the shrinking of the nanowire core to sizes of the order of $10 \mathrm{~nm}$ or below. Moreover, we expect the existence of a relatively high density of interface states at the border between the crystalline core and the amorphous shell. In the case where the density of these electronic states is high enough, they can induce the complete depletion of the crystalline core [36]. In order to use these nanowires in electronic applications, it is necessary to develop a process to etch the amorphous shell and passivate the crystalline core.

In conclusion, we have presented a detailed study on the spatial dependence of the structure of indium-catalyzed germanium nanowires. Spatially resolved confocal Raman spectroscopy has shown variations in the structure along the nanowire axis. A variation in the crystalline fraction is observed, along with a change in the diameter of the crystalline core. The measurements are consistent with detailed TEM investigations and could explain the high resistivity of the nanowires. These results show the importance of spatially resolved Raman spectroscopy for the correlation of the structural and electrical properties.

\section{Acknowledgments}

The authors gratefully thank G Abstreiter and A W Holleitner for experimental support and fruitful discussions. Funding from the Marie Curie Excellence Grant SENFED and the DFG excellence initiative Nanosystems Initiative Munich (NIM) is much appreciated. The authors would like to thank the Serveis Cientificotècnics in Universitat de Barcelona for the TEM facilities. JA acknowledges the funding from CSIC in the PIE project NEAMAN.

\section{References}

[1] Zheng G F, Patolsky F, Cui Y, Wang W U and Lieber C M 2005 Nat. Biotech. 231294

[2] Hernandez-Ramirez F, Tarancon A, Casals O, Arbiol J, Romano-Rodriguez A and Morante J R 2007 Sensors Actuators B 1213

[3] Lu W, Xiang J, Timko B P, Wu Y and Lieber C M 2005 Proc. Natl Acad. Sci. 10210046

Knoch J, Riess W and Appenzeller J 2008 IEEE Electron. Devices 29372

[4] Kayes B M, Atwater H A and Lewis N S 2005 J. Appl. Phys. 97114302

Colombo C, Heiss M, Graetzel M and Fontcuberta i Morral A 2009 Appl. Phys. Lett. 94173108

Tian B, Kempa T J and Lieber C M 2009 Chem. Soc. Rev. 3816

[5] Cui Y and Lieber C M 2001 Science 291851

[6] Bjork M T, Ohlsson B J, Sass T, Persson A I, Thelander C, Magnusson M H, Deppert K, Wallenberg L R and Samuelson L 2002 Appl. Phys. Lett. 801058

[7] De Franceschi S, van Dam J A, Bakkers E P A M, Feiner L F, Gurevich L and Kouwenhoven L P 2003 Appl. Phys. Lett. 83344

[8] Minot E D, Kelkensberg F, van Kouwen M, van Dam J A, Kouwenhoven L P, Zwiller V, Borgstrom M T, Wunnicke O, Verheijen M A and Bakkers E P A M 2007 Nano Lett. 7367

[9] Lauhon L J, Gudiksen M S, Wang C L and Lieber C M 2002 Nature 4206911

[10] Wang D W, Wang Q, Javey A, Tu R, Dai H J, Kim H, McIntyre P C, Krishnamohan T and Saraswat K C 2003 Appl. Phys. Lett. 832432

[11] Kamins T I, Li X and Kamins R S 2004 Nano Lett. 4503

[12] Jagannathan H, Deal M, Nishi Y, Woodruff J, Chidsey C and McIntyre P C 2006 J. Appl. Phys. 100024318

[13] Shimizu T, Zhang Z, Shingubara S, Senz S and Gosele U 2009 Nano Lett. 91523

[14] Kamins T 2007 Semiconductor Nanowires for Electronics and Sensors vol 397 HE-Heraeus-Seminar Semiconductiong Nanowires: Physics, Materials and Devices

[15] Kamins T I, Williams R S, Chen Y, Chang Y L and Chang Y A 2000 Appl. Phys. Lett. 76562

[16] Wang Y W, Schmidt V, Senz S and Goesele U 2006 Nat. Nanotechnol. 1186

[17] Colombo C, Spirkoska D, Frimmer M, Abstreiter G and Fontcuberta i Morral A 2008 Phys. Rev. B 77155326

[18] Zardo I et al 2009 Nanotechnology 20155602

[19] Xiang Y et al 2009 Nanotechnology 20245608

[20] Xiang Y, Cao L Y, Arbiol J, Brongersma M L and Fontcuberta i Morral A 2009 Appl. Phys. Lett. 94163101 
[21] Claeys C and Simoen E 2007 Germanium-Based Technologies. From Materials to Devices (Amsterdam: Elsevier)

[22] Fontcuberta i Morral A, Arbiol J, Prades J D, Cirera A and Morante J R 2007 Adv. Mater. 191347

[23] Gupta R, Xiong Q, Adu C K, Kim U J and Eklund P C 2003 Nano Lett. 3627

[24] Jalilian R, Sumanasekera G U, Chandrasekharan H and Sunkara M K 2006 Phys. Rev. B 74155421

[25] Spirkoska D, Abstreiter G and Fontcuberta i Morral A 2008 Nanotechnology 19435704

[26] Bermejo D and Cardona M 1979 J. Non-Cryst. Solids 32405

[27] Voutsas A T, Hatalis M K, Boyce J and Chiang A 1995 J. Appl. Phys. 786999

[28] Veprek S, Sarott F A and Iqbal Z 1987 Phys. Rev. B 363344
[29] Bustarret E, Hachicha M A and Brunel M 1988 Appl. Phys. Lett. 521675

[30] Brodsky M H, Cardona M and Cuorno J J 1977 Phys. Rev. B 163556

[31] Richter H, Wang Z P and Ley L 1981 Solid State Commun. 39625

[32] Campbell I H and Fauchet P M 1986 Solid State Commun. 58739

[33] Zhang Y F, Tang Y H, Wang N, Lee C S, Bello I and Lee S T 2000 Phys. Rev. B 614518

[34] Cronin S B, Lin Y M, Rabin O, Black M R, Ying J Y, Dresselhaus M S, Gai P L, Minet J P and Issi J P 2002 Nanotechnology 13653

[35] Keithley 6487 voltage source and picoammeter

[36] Hanrath T and Korgel B A 2005 J. Phys. Chem. B 1095518 